\title{
Sharing knowledge in knowledge-intensive firms
}

\author{
Juani Swart and Nicholas Kinnie, University of Bath \\ Human Resource Management Journal, Vol 13 No 2, 2003, pages 60-75
}

\begin{abstract}
Knowledge-intensive firms need to share knowledge held by employees if they are to gain the most from their intellectual capital and compete effectively in the marketplace. Sharing and integrating knowledge within the organisation depends partly on building social capital. However, there are obstacles to this integration because knowledge is often distributed throughout the organisation. This article draws on a detailed study of a single case to examine the ways in which HR policies and processes contribute to overcoming these barriers to sharing knowledge. We highlight the role of HR policies and processes as facilitators of the social capital that interconnects knowledge by focusing on the needs of knowledge workers.
\end{abstract}

Contact: Juani Swart, Work and Employment Research Centre (WERC), School of Management, University of Bath, Bath BA2 7AY

uccessful knowledge-intensive firms (KIFs) gain competitive advantage from the human and social capital which make up their unique trading assets (Alvesson, 2001; Frenkel et al, 1999; Lei et al, 1999; Newell et al, 2001; Purvis et al, 2001; Starbuck, 1992). Human capital includes individual tacit and explicit knowledge (Nelson and Winter, 1982) brought into the organisation through its knowledge workers, while social capital refers to knowledge that is embedded within the organisational relationships and routines. These forms of capital therefore comprise the knowledge and skills of individual employees and the relationships between these employees. Consequently, sharing knowledge between client projects, for example, becomes critical to the performance of the KIF.

There are, however, obstacles to sharing and growing knowledge which is typically distributed throughout the organisation and embedded within its routines (Tsoukas, 1996). This article focuses on the management of what we refer to as the tension between the integration and distribution of knowledge. In particular, it asks how the management of HRM can contribute to overcoming the barriers to sharing knowledge in KIFs. In doing so it adds to the growing research into the HR practices of these organisations (Mehta, 2001; Robertson and O'Malley Hammersley, 2000; Schubert and Ginsburg, 2000; Weigand et al, 2000).

We take a grounded theory approach to address this question using data from a continuing empirical study of growing KIFS, characterised by a case-based approach. Through detailed analysis of one of these cases we highlight the role of HR policies and, more importantly, HR processes as facilitators of social capital. These processes comprise social supports that interconnect knowledge within a distributed knowledge system by focusing on the needs of individual knowledge workers (Oldham and Cummings, 1996). In particular, the case illustrates how an HR advantage (Boxall and Steeneveld, 1999; Boxall and Purcell, 2003) and an intellectual capital advantage can be gained by ensuring that the processes for managing people provide close support for the processes of knowledge sharing. 
The presentation and analysis of our case-study data form the core of this article. However, we first need to establish the distinctive characteristics of KIFs and to explain how we conducted the study.

\section{DEFINING KNOWLEDGE-INTENSIVE FIRMS}

The phrase 'knowledge-intensive' can be used in at least three contexts: knowledgeintensive work, knowledge workers and knowledge-intensive firms. Furthermore, the distinction between knowledge-intensive and non/less knowledge-intensive organisations or work is not self-evident (Alvesson, 2001). All types of work and work organisations appear to involve knowledge: employees need 'know-what' and 'knowhow' in order for any firm to create sustainable competitive advantage (Quinn, 1992).

The category of knowledge-intensive firms (Alvesson, 1995; Robertson and Swan, 1998; Starbuck, 1992) refers to those where most work is said to be of an intellectual nature and where well-educated, qualified employees form the major part of the workforce (Alvesson, 2000). Furthermore, the company claims to produce qualified products and/or services (Alvesson, 2000, 2001). Typical examples of KIFs include law and accounting firms, management, engineering and computer consultancy companies, advertising agencies, research and development units and high-tech companies.

The distinctive characteristics of KIFs are examined by considering the type of input or capital, the type of work and the industry characteristics.

\section{Knowledge intensity and type of input or capital}

The key resource in KIFs is often referred to as human capital or the intellectual material - knowledge, information, intellectual property and experience - that can be put to use to create wealth (Stewart, 1997). Starbuck (1992) suggests that 'knowledgeintensive' can be applied to firms in which knowledge has more importance than other inputs, and human capital, as opposed to physical or financial capital, dominates. As the chief executive in our case study put it:

We are a people company. We make money through the knowledge of our people and if we don't know how to successfully manage people then we would simply die as a company.

The reliance on human capital, as opposed to physical capital, in order to compete in the marketplace is regarded as a key differentiator of KIFs. Support for this approach can be found in the accounting and finance literature through the use of Tobin's q. This ratio measures the relationship between a company's market value and its replacement value or its physical assets (Sveiby, 1997). Companies in the software industry tend to have a Tobin's q ratio of 7.00, whereas more traditional firms, such as within the steel industry, have a Tobin's q ratio of nearly 1.00. The value of intellectual capital and intangible assets is therefore reflected in a high Tobin's q and is useful in selecting KIFs.

Alvesson (2001) defines a KIF as a company where the majority of employees are well qualified, while Bontis (1998) sees the quality of human capital as a source of innovation and strategic renewal. Thus, employee skills are central to the creation of competitive advantage and, indeed, to the survival of the organisation when market conditions are tough. However, it is not only the presence of human capital that it is important in defining KIFs: it is also the way in which it is applied that makes these organisations distinctive. 


\section{Applying human capital}

Starbuck (1992) argues that it is the application of expertise which makes an important contribution in KIFs, while others draw attention to the capacity to solve complex problems through creative and innovative solutions (Hedberg, 1990; Sveiby, 1997). The application of human capital highlights two important issues. First, creative jobs, such as in advertising, are not necessarily knowledge-intensive if they do not involve intricate problem solution. Secondly, standardised work is not regarded as knowledgeintensive, even if it requires high levels of intellectual capital. For example, the making of flutes (Yanow, 1999) requires knowledge about flute-making but its repetitive nature, which leads to skills being embodied, does not qualify as knowledge-intensive. In other words, doing a clever thing over and over does not mean that it is knowledge-intensive.

It can be argued that the output of the 'exceptional contribution that intellectual capital makes' is questionable. Often the output is intangible - for example, consulting advice - and its quality is difficult to determine. Whether a solution is 'good' or not is often determined by factors external to the solution itself: for example, changing market forces, the interpretation of the clients buying the solution and the degree of trust that the sellers of the solution inspire. This ambiguity surrounding outcomes is central to KIFs but presents a very real barrier to measuring performance outcomes. For this reason we focus on work processes - novel, complex and involving problem solution - as indicators of KIFs.

We therefore extend our definition of KIFs and describe these organisations as knowledge-intensive, not only because income is generated through intangible assets but also because of the nature of the deployment of the knowledge held. In other words, our criteria of knowledge-intensive firms extend to the enactment of tacit knowledge in novel circumstances. Within this criteria our research resonates with that of Lei et al (1999) who put renewed emphasis on the importance of innovation, initiative and competence building in knowledge-intensive organisations. It is within this context that tacit knowledge is the prime driver for value creation.

\section{Industry or firm-specific?}

Reference is often made to a particular industry being knowledge-intensive: for example, biotechnology or management consulting. It is, however, important to recognise that big differences could exist between firms within a particular industry. Large, well-known management consultants have clear and set procedures, and some of the more popular consultancy models have been published (Doorewaard and Meihuizen, 2000). In contrast, smaller management consultancies tend to focus on bespoke services and solutions.

Consequently, it is unwise to define a particular industry as knowledge-intensive, and the unique knowledge-intensive criteria presented here need to be applied to individual organisations. We therefore define KIFs as the organisations within a knowledge economy (Drucker, 1993) that employ highly skilled individuals and therefore create market value through the application of knowledge to novel, complex client demands.

More precisely, we define a KIF in terms of their emphasis on:

1 The nature and quality of their highly skilled human capital;

2 The work processes that create market value through knowledge; and

3 The deployment of the knowledge involving innovation, initiative and competence building in the provision of bespoke services. 
There are, however, two further characteristics of KIFs on which we wish to focus. First, KIFs often have different growth patterns when compared with more traditional organisations. Typically they have been borne out of an innovative idea that addresses a niche market need. Given the opportunity that technology affords these organisations in terms of global marketing, they tend to grow fast in number of employees, although the use of sub-contractors and other alternative work arrangements means that the directly employed workforce will not always grow quickly. In our research, however, we focus on KIFs that are successful in the market and are faced with organic growth challenges. This draws attention to fundamental challenges in knowledge-based competition: how to attract, motivate and develop workers with critical skills and aptitudes (Boxall and Purcell, 2000).

Secondly, we draw attention to the importance of the market relations that KIFs encounter. Many of these firms are engaged in business-to-business relationships with a relatively small number of clients, rather than selling their services directly to the end user. Supplying services to a few clients, especially when contracts are short, can create a highly unpredictable and unstable market environment. Moreover, these contracts for services are difficult to value, thereby creating further pressures from financial markets, especially when the organisation has plc status. These sources of environmental instability ensure that the problems of sharing knowledge become even more fundamental to organisational success.

\section{Defining knowledge}

Building on the work of Tsoukas (1996), we see the firm as a 'distributed knowledge system'. This social constructionist perspective argues that knowledge is not a given or external to the individual but is constructed through discourse and the patterns of interrelations between employees. Consequently, each knowledge worker builds, through social practice, a representation of how to act and who to engage with in action in complex, novel situations (Swart, 2000). Knowledge and practice are intertwined and, as practice is distributed, so, too, is the knowledge that is embedded within the practice.

The critical knowledge type that is distributed throughout the organisation is tacit knowledge. This phrase was first coined by Polanyi (1966) and used to describe a form of knowledge that cannot be explicated and that is embodied through practice. At the heart of this concept is the notion of tacit 'knowing': here, the outcome of action is the focal, or proximal, point and the doing (achieving the outcome) is characterised as a distal process. Through practice, the attention of the doer is focused on the outcome rather than the means of achieving the outcome.

We distinguish here between:

Practice-based tacit knowledge This is is driven by the culture in the KIF and is embodied, for example, in the application of a software code, ie knowing the short-cuts around the code and how to apply it in a way that adds value to the customer.

Technical tacit knowledge This is embedded in knowing the code in the first place. This form of tacit knowledge has been equated with explicit knowledge in previous research but our research participants indicated that it was impossible to write down or capture their technical knowledge; it could be taught only through shared practice (learning-by-doing with others).

These two forms of tacit knowledge are therefore intertwined, and technical knowledge cannot be developed without practice-based tacit knowledge. It is also 
through the integration of these forms of knowledge that critical skills are developed for a base of competition in KIFs.

However, the critical skills and knowledge of employees and their social practices may become localised within a project team or a more informal community-of-practice (Wenger, 2000; Yanow, 1999) within the organisation. It is essential for the success of the organisation that this knowledge is integrated between the different pockets and shared throughout. Connections need to be made between the potentially disparate parts of the organisation if the knowledge is to be developed. We explore how this tension between the distribution and the sharing of knowledge is managed by drawing on a case study of a growing knowledge-intensive firm. We pay particular attention to the role of HR practices and processes in establishing interconnections within a distributed knowledge system.

\section{METHODOLOGY}

Our data are drawn from a case study conducted as part of a wider project, funded by the CIPD, which explores the relationship between business performance and HR practices in six small to medium-sized growing KIFs. This research is therefore casebased and characterised by a grounded theory methodology (Strauss and Corbin, 1994; Partington, 2000): data gathered and analysed in the earlier stages of the research are used to develop further data gathering.

We interviewed all members of the organisation across three levels: first at director level, then at line/project manager level and finally at the employee level. At the first level we used unstructured interviews to gain an insight into the historical context, pertinent challenges faced by the organisation and its market position, as well as the strategic decisions that were taken. These data were then analysed via the open-coding procedure, grouped into secondary or axial codes and represented here as themes.

The aim of the semi-structured line/project manager interviews was to ascertain how HR policies, procedures and practices operated within each organisation. The structured employee interviews focused on how the employees perceived the impact of the HR practices. All the interviews were conducted on-site and each lasted approximately 90 minutes. Questions were focused on HR practices that were identified following a thorough literature review. The analysis of data gathered was similar to that of the director-level interviews, identifying key themes within the broader implementation of HR practices. Using our theoretical foundation of the characteristics of a distributed knowledge system we conducted a thematic analysis to identify how our case organisation addressed the distributed nature of knowledge.

\section{The case of SoftWareCo}

SoftWareCo employed 46 people and was located on two sites in the same town in the south-west of England. Founded in 1986, it was the brainchild of three software engineers who wanted to focus their commercial work on bespoke software development in embedded systems. A conscious decision was made at the outset by the owners to grow their business organically and to value quality of life. As the managing director put it:

The quality of life is the most important thing for us. You have to remember our people are all we have. We don't have products - only people.

A flat structure with only three levels - directors, senior software engineer (SSE) and software engineer (SE) - was maintained through periods of growth. Most of the 
employees were software engineers, with hardly any employees in traditional or specialist functions such as finance, HRM or marketing. The majority of the workforce was young and male, and there was a strong belief that women would not be interested or competent at the type of work undertaken. Nonetheless there was a strong drive to involve families in social and work events, and the integration of family and work life was seen as a key practice that motivated and retained staff.

The market focus also remained on bespoke software development, and commercial efforts were directed towards the development of modules, subcomponents and hardware-software interfaces for multinational clients. A clear strategy was to counter the current trend in software development and to steer away from the product route because it was believed that this would have a devastating effect on the organisation's culture and staff retention. Its specific suite of software services meant that it was difficult to recruit the right skills in the current labour market, and this was intensified by universities taking a commercial approach, rather than technical specialist, in their education of software engineers. Finally, the organisation occupied a dominant position within a niche market, with its main competitors being independent consultants.

\section{Operational differences}

The organisational structure and operational process within this case organisation differed somewhat from other KIFs. SoftWareCo did not have traditional/specialist departments and functions; instead, the key operational processes were distributed across various committees and teams. It could be argued that all small firms would have these distributed and informal processes. However, in our sample of six KIFs most organisations followed traditional organisational forms based on hierarchy and specialism, and had standardised HR policies which would not be out of place in a manufacturing company. We regard this particular characteristic as a unique operational quality, and will demonstrate how this distributed and socially embedded way in which both knowledge and people are managed enables knowledge sharing.

SoftWareCo's key HR practices evolved through social practices and were distributed across the following three sub-structures (see Figure 1 overleaf):

- The committee structure, handling what would otherwise be the more traditional HR function, with all employees being members of at least two committees;

- The mentoring structure, where each senior software engineer took responsibility for the resource development of a more junior engineer on another project; and

- The project structure, which was the main organisational structure for the organisation of bespoke development work.

Most enabling ${ }^{1}$ HR practices and the core HR roles were divided between several positions (directors and senior software engineers). HR practices were formalised through the committee structure, where suggestions from directors, senior software engineers and software engineers were published on the intranet, discussed at a formal gathering and then approved by directors at their monthly meeting. This forum did afford the space for any disagreements to be voiced, as well as for current practices to be challenged. However, we found more evidence of the acceptance and continued practice of people management processes than criticism or change. The strong socialised nature of the practices may have encouraged acceptance rather than criticism. On the other hand, knowledge management issues, such as the use of an intranet to communicate skill development, were frequently challenged and debated in technology chat rooms, and several changes were suggested. 
FIGURE 1 HR practices at SoftWareCo

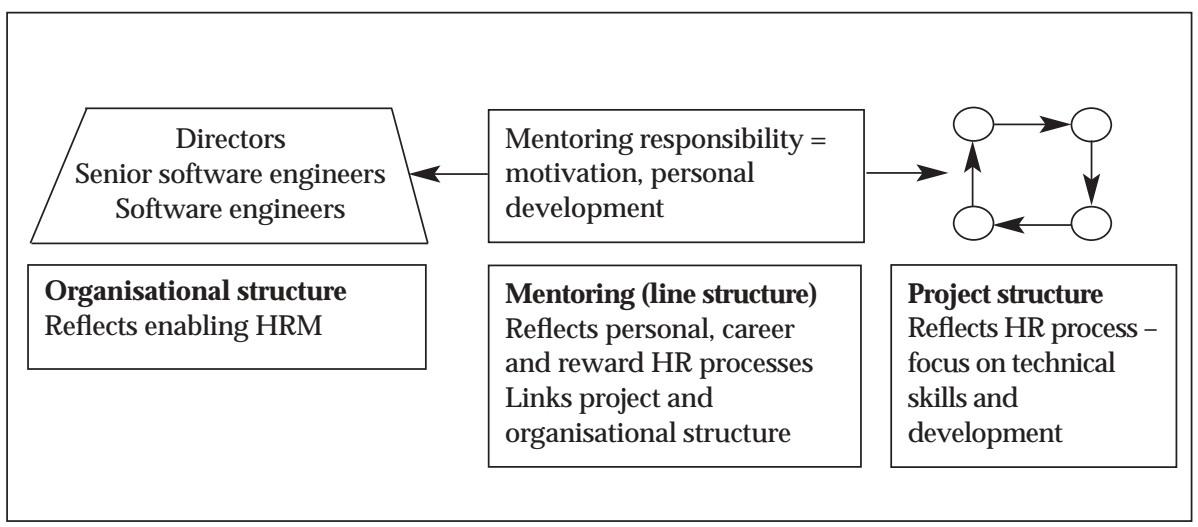

Although the enabling HR practices were more formalised and the responsibility split between directors and senior software engineers, the heart of the HR processes were a lot more informal and responsibility for them rested with mentors and project managers. The mentoring scheme, where each senior software engineer had two or three protégés (who did not report to them) was key to this. Mentors, acting in a line management capacity, were responsible for the implementation of HR processes focusing on personal and career development via the performance management system. Project managers took a leading developmental role in a technical skill enhancement capacity by working on project skills which were taught by 'working together'. Within this approach a director could be reporting to a software engineer on a project and consequently be trained by a younger and more junior member of staff.

It may be expected that these unusual reporting lines could cause tension and disagreement; yet, when questioned about the political dynamics of such as system, our participants remarked that it added excitement to their involvement in a project. Another clear theme that was identified in this form of development was that the more senior software engineers felt that there was 'something in it for them', and that they were able to demonstrate that they were familiar with the latest technology and software development approaches. Unlike other organisations in our research, knowing the latest languages and approaches was not seen as a means of leaving the organisation but rather as a tool to keep clients and to complete projects in a shorter period of time. Here, knowledge sharing and people development, via the mentor, intertwined to create a client benefit.

The mentoring committee was one of many in the organisation, each enjoying a high degree of autonomy, and high levels of participation in decision-making were facilitated through these structures. The developmental process was strongly linked to reward, although remuneration was the one issue that was dealt with only at director level. Directors jointly determined pay levels and made decisions about increases based on the recommendations made by the mentors as the outcome of performance appraisal discussions. The implementation of skill enhancement rested with mentors, where responsibility was taken for the development of skills of employees who did not report directly to them. This developmental process was then integrated further into the mentoring committee, where the mentors themselves had the opportunity to develop.

The project structure was seen as the main sub-structure that integrated other operational processes. Work was allocated according to client project teams, and 
resource allocation was then driven by the demand for skills within these teams. The dominance of the project teams presented the danger that practices and knowledge could become localised and fragmented. However, the other sub-structures that spanned the project boundaries ensured that social practices were distributed and that knowledge was managed within and between project teams.

Furthermore, the sub-structures represented the vehicles through which all HR practices evolved in this organisation: it was through suggestions made in committees or social practice that was shared throughout the organisation that specific HR processes were shaped. The employees themselves generated the manner in which people were managed, and it was this ownership that then drove coherent practice. Unlike other KIFs, where practices may divert from policies or where employees feel negative towards a performance appraisal scheme, there was less discrepancy between espoused theories and theories-in-use at SoftWareCo.

\section{HR practices in SoftWareCo}

SoftWareCo operated within a very tight labour market and knowledge workers within this industry preferred to work for large well-known organisations along the so-called 'M4 corridor', stretching west of London towards Swindon. The location of this company therefore hindered the attraction of the best talent in the external labour market. Furthermore, the market specialisation of the organisation was directed at a small niche of software engineering skills, with few students specialising in embedded software. However, within the tight labour market, SoftWareCo had extremely strict selection criteria. Technical ability was not considered the most important element and it was the company's culture that led the recruitment process. As the senior software engineer who shared the responsibility for recruitment remarked:

I think of it as inviting someone to a party. You know, sometimes you invite people who you want to come along - not necessarily those who deserve to come along.

The senior software engineer responsible for recruitment identified candidates for recruitment by using his widely known networks within the industry. Both pre and post-experience candidates would be approached for interviews and, at this stage, it was is normally taken for granted that the employee would have adequate technical tacit knowledge. This was due to the high level of technical specialisation needed to be approached for an interview and also the small size of the network within which these engineers operated; technically competent employees would be well-known within their industry.

This senior software engineer and some of the directors then conducted interviews, which were very informal and took on the format of a 'communication of ideas or solutions' to a particular software problem. The ability to generate innovative thought and then to communicate these ideas were important criteria in the selection process. Recruits needed to show how they would share their innovative ideas and cuttingedge know-how within a project team. This formed the basis on which the 'knowledge network' worked (as one senior software engineer referred to it).

Knowledge networks were 'built up' through socialised practices which were embedded through high retention rates. A key factor in the attraction and retention of senior software engineers and software engineers was the nature of the work conducted by the organisation. Employees got the opportunity to work on cutting-edge technology and to ensure that their skills remained at the forefront of this fast-changing industry. As one senior software engineer remarked: 
If you ask people here what is the best thing about this place, besides that atmosphere, people will say it is the interesting work. You are always learning something new; you will never get bored.

The challenging work appeared to be more critical to retention than the salary levels, as SoftWareCo did not pay better than its competitors in the local area. Employees were more focused on the quality of life that the small, innovative organisation could offer them. A senior software engineer felt that 'you can only pay people more to keep them, but here we do not have a problem with that - the people enjoy the type of work.' The development of skills and the opportunity to apply skills were therefore seen as a unique form of reward.

The resource creation process (Tsoukas, 1996) was driven by a culture of innovation (self-teaching and employee-driven), knowledge sharing within projects and learning between projects. The project structure was consequently the main vehicle for the formal and informal development of resources. Within the project structure the most competent person would manage a project; this was often a software engineer or a student engineer and a junior member of staff could, as a result, have a director reporting to them.

The most competent person would be decided on the basis of prior experience. In other more traditional work environments this might result in disagreements. However, in SoftWareCo there were several strands of technical skill, with certain employees specialising in each of these strands. Technical experts were also recognised through informal networks and by working together across various project teams. Everyone was given the opportunity to acquire management skills, and those who did not want to take this route in their careers had the opportunity to be project engineers (developing) and advisers (working on another project but giving advice in their area of specialisation).

Development was not only driven through informal and embedded processes but was also formalised and linked to project and software development outcomes through the performance management system. SoftWareCo had a complex performance management process which evolved through suggestions and practice of the software engineers. This review process, which took place over and above the continuous reviews on a project, comprised two stages.

First, project performance reviews, conducted by the project manager, focused on project efficiency and the employee's technical ability within the project. Here, three forms of review were implemented: a self-appraisal focusing on jointly set objectives, project performance, technical ability, self-management, team contribution and customer satisfaction; a peer review on the same dimensions; and a management review of the employee. A direct link was established between project performance reviews and annual increases.

Secondly, bi-annual performance reviews focused on employee development and spanned project boundaries, thereby ensuring that practice was shared throughout the organisation. These reviews were conducted by mentors who collated all project performance reviews, completed a protégé appraisal on overall performance areas and conducted a performance discussion.

The project structure was also fluid, and people were rotated between projects to maintain a level of interesting work and to broaden their technical skill range. On average, an employee would move on to a new project every 18 months, and rotation between sub-projects within the larger project, originating from specific client requests, was more frequent. A project manager (not always a senior software engineer) managed up to four sub-groups and conducted development work on one project: 
Here we don't have people who do only project management - and quite honestly with the kind of work we do [bespoke] you will never find someone who would want to do only project management work. It would be far too boring, and we want to keep our skills at the cutting edge.

'Learning from the master' was highly valued within the project structure and, if the 'master' was not within the project, then it was the responsibility of the project manager to point the particular employee 'in the right direction' to develop a specific technical skill. External sources such as the internet were also relied on heavily for the development of code, and university networks were often consulted when employees were faced with challenging customer demands. Boundaries between projects and organisations were fluid in order to encourage resource development.

Finally, learning was regarded so highly in the organisation that high performing employees were often rewarded with training days and were encouraged to select training unrelated to their core function in the organisation. Development of non-core skills was normally conducted off-site through a different provider. Employees were then encouraged to share informally what they had learnt at a weekly meeting or on their intranet page. Examples of training were learning a different language, art courses or technical courses that were related to the organisation but not central to its current market positioning. The use of 'redundant time' for the development of resources was therefore highly valued and accounted for in the formal appraisal procedure.

\section{DISCUSSION}

Although a project-based structure, which facilitated the distribution of knowledge, was used in SoftWareCo, various mechanisms were employed to share or connect the knowledge. These mechanisms meant that employees were familiar with 'who knows what', 'who is working on what' and 'who to ask when particular questions are raised'. Knowledge was shared by cross-functional meetings, voluntary membership of committees, conversations via newsgroups, participation in frequent social events and the induction process, as well as the intranet. Mechanisms were sufficiently strong to overcome any problems associated with the company being based on two sites. Employees were also encouraged to build networks with clients and the local community and, as a result, the notion of 'connectivity' was taken beyond the boundaries of the organisation.

Both cultural and technical knowledge were therefore connected throughout the organisation. These forms of connectivity were created through high levels of participation in decision-making and by maintaining a central focus of bespoke development. Such processes may have been aided by the homogeneity in the organisation, which was largely brought about by a choice to grow relatively slowly and to employ mainly specialists.

The integration of distributed knowledge was therefore underpinned by particular recruitment and selection choices and employee development and participation strategies. It was also evident in the unique manner in which these practices were implemented, thereby developing a context of HR practices as a form of participation (see Figure 1). We found that the combination of this particular set of HR practices created specific conditions for the establishment of 'knowledge networks' (see Figure 2 overleaf).

First, there was a strong cultural drive behind the recruitment process: prospective employees were recruited on the basis that they 'fit' within the organisation. A 
FIGURE 2 HR practices and processes and their impact on knowledge sharing

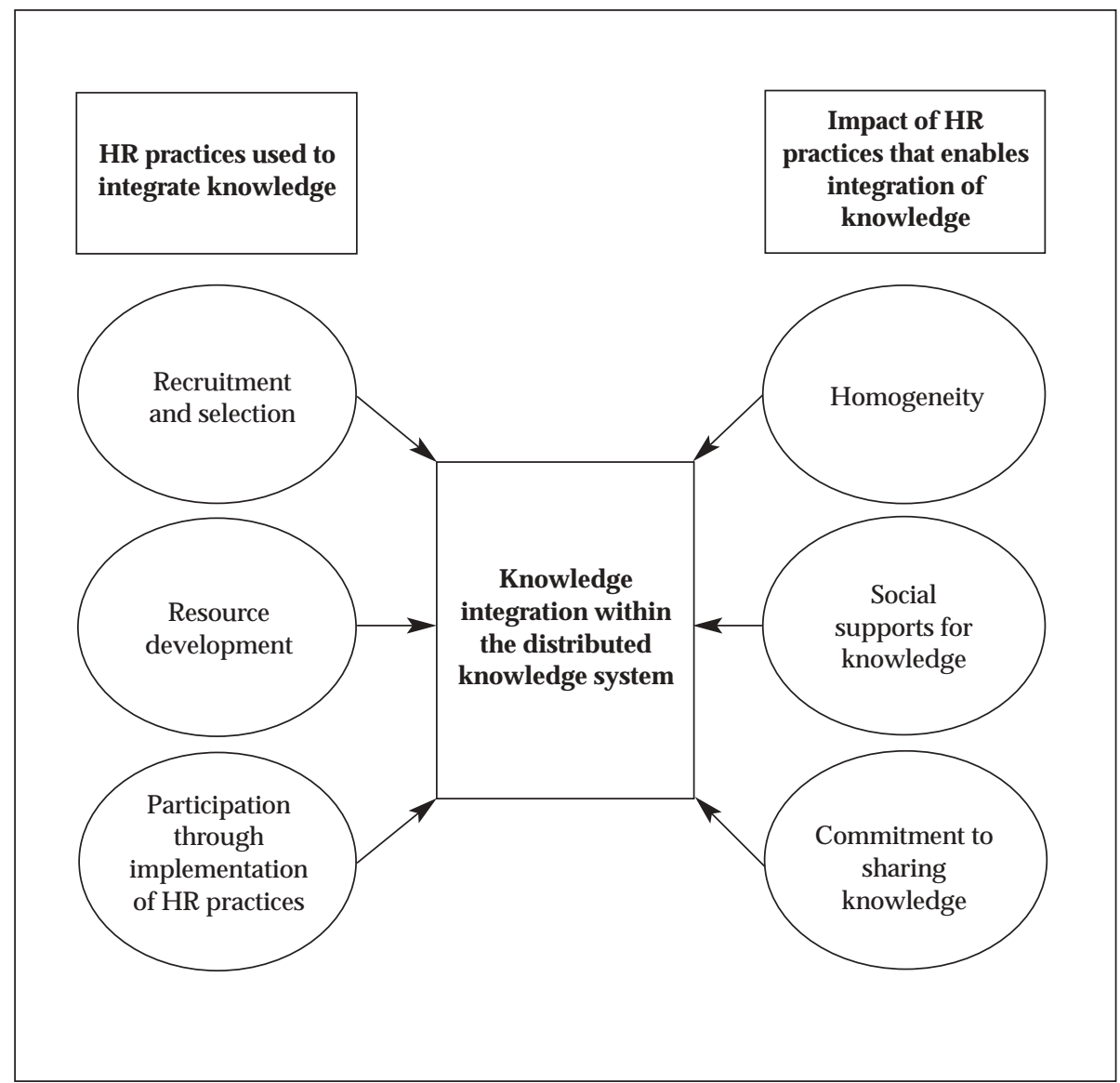

participant highlighted above that it was like inviting someone to a party, suggesting a notion to select according to family/social model, ie inviting those whom we would like to attend, rather than those who deserve to attend. This selection model enabled connections to be made within the distributed knowledge system by establishing shared mental models (by employing mainly software engineers) and a continued commitment to the sharing of knowledge. The latter was particularly evident in the extent to which attention was paid to verbal and symbolic communication: for example, the ability to draw conceptual models at the selection phase. Once again, these HR practices were focused on the sharing of knowledge and the provision of social supports for interconnecting various stakeholders in the knowledge sharing process.

Employee development was the second key process through which the distributed knowledge system became interconnected. The approach to development taken in SoftWareCo was characterised by cross-boundary learning, work-based learning (Raelin, 1997) and learning by doing (Lave and Wenger, 1991), depicting the sharing of knowledge through real-time problems and the implementation of learning across boundaries. As a participant remarked:

We all depend on one another; we pick one another's brains all the time. 
The design of the development process was therefore focused on integrating communities and 'drawing together' the tremendous potential of distributed knowledge within the organisation. The particular design of learning across projects through rotation between various client projects supported the notion of sharing knowledge further: fluid boundaries were evident in the manner in which HR practices were implemented.

The implementation of HR practices through key sub-structures facilitated the sharing of knowledge. Mentoring processes ensured that knowledge remained interconnected by appointing mentors who did not have functional/technical responsibilities, and committees spanned fluid project boundaries. These processes were cross-boundary not only in their origin but also in their implementation, and comprised a form through which the sharing of knowledge was controlled, reflecting the culturally driven nature of these practices.

HR practices were embedded in organisational routines and had become part of 'the way things are done', thereby encouraging an informal yet standardised approach to the management of knowledge workers, as well as the sharing of knowledge. The committee structure in particular created several communities-of-practice across the organisation that integrated practice. At the heart of these was a strong sense of identification with the organisation's culture, history and core capabilities. These vehicles for the implementation of $\mathrm{HR}$ practices were socially driven and encouraged a social/integrative approach to the practices that created a competitive advantage: the development of cutting-edge bespoke software.

A strong sense of social consensus governed the organisation; high degrees of participation were made possible through strong cultural controls. Practices were also developed informally and became embedded through continued practice. For instance, the focus of the recruitment process as 'inviting someone to a party' strengthened this consensus and created an environment where common frames of understanding were established and knowledge sharing was facilitated. Knowledge integration was also encouraged through the approaches to development, which relied strongly on culturally driven forms of engagement and agreement.

In addition to this, the HR practices addressed an issue which was at the heart of the tension between the distribution and sharing of knowledge: not only did they pay attention to the needs of the organisations but also, critically, to the needs of individual knowledge workers. Indeed, the HR practices in SoftWareCo were designed to stimulate the development of knowledge for employees which was key to their satisfaction and commitment, and the sharing of that knowledge which was central to organisational success. This related directly to retention rates - regarded as critical in knowledge-based competition (Boxall and Purcell, 2000). As one of the employees noted: 'There is a strong knowledge base and everyone is keen to help out throughout the project.' The knowledge workers themselves derived benefits from an integrative knowledge system, and the process of developing these interconnections became embedded in the HR practices.

This interconnection is central to the growth and success of KIFs, as such organisations are highly dependent on their principal assets - their employees. Moreover, the ability to 'grow knowledge' depends greatly on the sharing of that knowledge between employees, which requires their co-operation. This places a premium on satisfying the needs of employees - the owners of intellectual capital. Indeed, the success of the organisation depends on managing these knowledge workers so that they are attracted to the organisation, they want to stay and they are highly motivated to perform well. 
These employees are likely to want challenging and interesting work with a high degree of autonomy. Complex, unique tasks are likely to provide them with the opportunity to apply and, crucially, develop their knowledge. They are looking not just to engage in the repetitive application of existing knowledge but they also want to develop their knowledge, and are most likely to do this if they are working in novel situations where problem solving is involved. This is also likely to involve a high degree of autonomy in their work, since this gives them the freedom to solve problems drawing on their own expertise and professional training. Indeed, it is this application of specialist knowledge to new situations that will provide a strong intrinsic incentive to these employees.

The various practices that are employed to address the needs of knowledge workers have been discussed and provide further insights into how SoftWareCo managed the tension between the distribution and sharing of knowledge. In addition to the practices designed to stimulate the connection of knowledge, the organisation also paid attention to the needs of its employees since they held the assets of the company, both collectively and individually. This was done by acknowledging that the development and sharing of knowledge was fundamental not only to organisational success but also to the needs of employees. The management of knowledge workers can therefore be regarded as a key variable in managing the tension between the distribution and the connection of knowledge.

\section{CONCLUSION}

We have seen how managers in SoftWareCo used HR practices and processes to support the sharing of knowledge within their organisation. These processes provided a series of social supports to overcome some of the obstacles to sharing knowledge which are commonly distributed throughout the organisation. In particular, these barriers can be crossed by paying attention to the needs of individual employees so that they see it as in their own interests to share knowledge. In effect, these HR processes are designed to create a complementarity between the needs of the employee for development and growth and the needs of the organisation to share knowledge. Such practices and processes contribute to the development of social capital (Nahapiet and Ghoshal, 1998) which promotes the conversion of human capital into intellectual capital. This is composed of organisation-specific knowledge and skills, as well as the outputs associated with these: for example, software programs, consultancy models and patents. Concentrating on the needs of employees provides the means of overcoming the competing pressures of the distribution and sharing of knowledge. Employees develop their own skills and knowledge by being given challenging tasks (the distribution of knowledge), but they also recognise that they need to share this knowledge within the organisation.

The approach to managing people in SoftWareCo was not simply an adaptation of the policies and practices employed in larger more traditional firms to take account of their knowledge-intensive characteristics, as some others seem to suggest (Robertson and O'Malley Hammersley, 2000). Distinctively, the approach demonstrates a mutually supportive focus on both knowledge-sharing processes and HR processes. Moreover, it seeks to embed both sets of processes so that they are taken for granted by all employees. The approaches to managing people and managing knowledge are mirror images of one another and share common assumptions. They emphasise the importance of the written HR policies and the processes involved in actually putting these into operation, and the significance of 
the formal mechanisms for exchanging explicit information and the more informal methods used for sharing tacit knowledge.

More generally, managers in SoftWareCo sought to gain complementary HR and intellectual capital advantage. As Boxall and Purcell (2003: 85-6) argue, HR advantage is gained through a combination of human capital advantage - in the form of both people and policies - and the HR and organisational process advantage needed to get the most out of this capital in a particular context. Similarly, gaining an intellectual capital advantage depends not just on the knowledge in the firm but also on the processes for connecting that knowledge. In particular, SoftWareCo achieved a knowledge-sharing process advantage which was supported closely by an HR process advantage. Moreover, it is this combination of these hard to imitate knowledge sharing and HR processes which is a genuine and robust source of competitive advantage.

SoftWareCo sought to generate a synergy between the processes of knowledge sharing and managing people in ways that were not present in some of the other cases we studied. Elsewhere, we saw examples of traditional hierarchical structures where teams operated in functional 'silos', with few mechanisms to share explicit knowledge, let alone tacit knowledge, between employees. Formal HR policies were either absent or took little account of the potential gap between the formal policy and the way this was actually implemented. Unlike the case of SoftWareCo, these policies were designed in ignorance of the opportunity to provide a stimulus to and support for knowledge sharing.

The process-based approach discussed here was not an inevitable consequence of the knowledge-intensive characteristics of SoftWareCo. Instead, it is the result of a series of conscious and unconscious choices which reflect and sustain 'the way things are done'. The choices made by senior managers and employees become evident in the way HR practices and processes evolved in SoftWareCo. This approach seeks to overcome the problem of sharing knowledge by applying a common perspective to the processes for managing people and managing knowledge which become embedded in the organisational routines. This combination of processes is designed to maximise the firm's intellectual capital to allow it to compete more effectively in the marketplace.

Although this case adopted a distinctive approach compared with a number of the other KIFs we studied, one key questions remains: to what extent can this approach be sustained if the organisation grows? In other words, was SoftWareCo's success in sharing knowledge and managing people simply a small company phenomenon? Although more data is needed, we suspect that it is not. Other research carried out in a much larger, successful KIF employing more than 400 people identified a very similar, mutually supporting set of people and knowledge management practices and processes. This organisation was also driven strongly by the values of its senior directors and managers, who adopted a processual perspective of the kind at SoftWareCo. Therefore, although organisational size is not necessarily an obstacle to sharing knowledge successfully, the people and knowledge management policies and processes will be of critical importance.

\section{Note}

1 'Enabling' refers to the basic HR practices that allow the organisation to 'play the game' or survive in the industry (Leonard, 1998), ie recruitment, selection, training, pay, etc. It is otherwise referred to as 'stakes' (Boxall and Steeneveld, 1999). 


\section{REFERENCES}

Alvesson, M. (1995). Management of Knowledge Intensive Companies, Berlin/New York: de Gruyter.

Alvesson, M. (2000). 'Social identity and the problem of loyalty in knowledge-intensive companies'. Journal of Management Studies, 37: 8, 1101-1123.

Alvesson, M. (2001). 'Knowledge work: ambiguity, image and identity'. Human Relations, 54: 7, 863-886.

Bontis, N. (1998). 'Intellectual capital: an exploratory study that develops measures and models'. Management Decision, 36: 2, 63-76.

Boxall, P., and Purcell, J. (2000). 'Strategic human resource management: where have we come from and where should we be going?' International Journal of Management Reviews, 2: 2, 183-203.

Boxall, P. and Purcell, J. (2003). Strategy and Human Resource Management, Basingstoke: Palgrave Macmillan.

Boxall, P., and Steeneveld, M. (1999). 'Human resource strategy and competitive advantage: a longitudinal study of engineering consultancies'. Journal of Management Studies, 36: 4, 443-63.

Currie, G. and Procter, S. (2001). 'Exploring the relationship between HR and middle managers'. Human Resource Management Journal, 11: 3, 53-69.

Doorewaard, H. and Meihuizen, H. E. (2000). 'Strategic performance options in professional service organisations'. Human Resource Management Journal, 10: 2, 39-57.

Drucker, P. (1993). Post-Capitalist Society, Oxford: Butterworth-Heinemann.

Frenkel, S. J., Korczynski, M., Shire, K. A. and Tam, M. (1999). On the Front Line; Organization of Work in the Information Economy, Ithaca, NY: Cornell UP.

Hedberg, B. (1990). 'Exit, voice and loyalty in knowledge-intensive firms'. Paper presented at the 10th annual international conference of the strategic management society, Stockholm, September.

Lave, J. and Wenger, E. (1991). Situated Learning: Legitimate Peripheral Participation, Cambridge: Cambridge University Press.

Lei, D., Slocum, J. W. and Pitts, R. A. (1999). 'Designing organizations for competitive advantage: the power of learning and unlearning'. Organizational Dynamics, Winter, 24-38.

Mehta, L. (2001). 'The World Bank and its emerging knowledge empire'. Human Organization, 60: 2, 189-196.

Nahapiet, J. and Ghoshal, S. (1998). 'Social capital, intellectual capital and the organisational advantage'. Academy of Management Review, 23: 2, 242-266.

Nelson, R. and Winter, S. G. (1982). An Evolutionary Theory of Economic Change, Cambridge, Mass: Harvard University Press.

Newell, S., Scarbrough, H. and Swan, J. (2001). 'From global knowledge management to internal electronic fences: contradictory outcomes of intranet development'. British Journal of Management, 12: 2, 97-112.

Oldham, G. R. and Cummings, A. (1996). 'Employee creativity: personal and contextual factors at work'. Academy of Management Journal, 39: 3, 607-634.

Partington, D. (2000). 'Building grounded theories of management action'. British Journal of Management, 11, 91-102.

Polanyi, M. (1966). The Tacit Dimension, London: Routledge and Kegan Paul.

Purvis, R. L., Sambamurthy, V. and Zmud, R. W. (2001). 'The assimilation of knowledge platforms in organizations: an empirical investigation'. Organization Science, 12: 2, 117-135. 
Quinn, J. B. (1992). Intelligent Enterprise, New York: The Free Press.

Raelin, J. A. (1997). 'A model of work-based learning'. Organization Science, 8: 6, 563- 578.

Robertson, M. and O'Malley Hammersley, G. (2000). 'Knowledge management practices within a knowledge-intensive firm: the significance of the people management dimension'. Journal of European Industrial Training, 24: 2/3/4, 241-253.

Robertson, M. and Swan, J. (1998). 'Modes of organizing in an expert consultancy: a case study of knowledge'. Organization, 5: 4, 543-64.

Schubert, P. and Ginsburg, M. (2000). 'Virtual communities of transaction: the role of personalization in electronic commerce'. Electronic Markets, 10: 1, 45-56.

Starbuck, W. H. (1992). 'Learning by knowledge-intensive firms'. Journal of Management Studies, 3: 4, 262-275.

Stewart, T. A. (1997). Intellectual Capital: The New Wealth of Organisations, New York: Doubleday.

Strauss, A. and Corbin, J. (1994). 'Grounded theory methodology: an overview' in Handbook of Qualitative Research. N. K. Denzin and Y. S. Lincoln (eds). London: Sage.

Sveiby, K. E. (1997). The New Organizational Wealth: Managing and Measuring KnowledgeBased Assets, New York: Berret-Keohler.

Swart, J. (2000). 'Collective tacit knowledge and self-awareness: an exploratory study'. Unpublished doctorate, University of Bath.

Tsoukas, H. (1996). 'The firm as a distributed knowledge system: a constructionist approach'. Strategic Management Journal, 17, 11-25.

Weigand, H., DeMoor, A. and VanDenHeuvel, W. J. (2000). 'Supporting the evolution of workflow patterns for virtual communities'. Electronic Markets, 10: 4, 264-271.

Wenger, E. (2000). 'Communities of practice and social learning systems'. Organization, 7: 2, 225-246.

Yanow, D. (1999). 'The languages of "organizational learning": a palimpsest of terms'. Proceedings from the Third International Conference on Organizational Learning, June, 1075-1086. 